\title{
Mitigation of transcultural clinical barriers in a health and wellness program: A transcultural caring approach for vulnerable migrant backstretch workers
}

\author{
Denise Nagle Bailey* \\ School of Nursing, La Salle University School of Nursing and Health Sciences, Philadelphia, Pennsylvania, USA
}

Received: September 17, 2014

DOI: $10.5430 /$ cns.v3n3p34

\author{
Accepted: September 23, $2014 \quad$ Online Published: April 18, 2015 \\ URL: http://dx.doi.org/10.5430/cns.v3n3p34
}

\begin{abstract}
This paper explores the mitigation of transcultural clinical barriers in a clinical setting where migrant backstretch workers receive health and wellness services from university faculty, staff, and nursing students at a nurse-led clinic located behind the stable area of a local thoroughbred racetrack. Caring for this vulnerable population is critical as these underserved individuals encounter many obstacles in the access of quality health care as a result of their marginalized status. Placing a heightened emphasis on providing culturally congruent care within the clinical setting and adopting a framework of transcultural caring principles using Ray's Transcultural Caring Dynamics in Nursing and Health-Care Model serve as a sound strategy to reduce cultural barriers and enhance the health of these workers who have inadequate or no healthcare coverage. This article examines the unique culture of migrant populations with a specific emphasis on migrant backstretch populations, and the health disparities that occur within this marginalized population. This article also addresses the importance of delivering clinical nursing services within the context of transcultural caring, applying the theoretical framework of Ray's Transcultural Caring Dynamics in Nursing and Health-Care to a nurse-led health and wellness program for migrant backstretch workers. It suggests clinical implications for nursing using a transcultural framework to improve the health status of this invisible population.
\end{abstract}

Key Words: Transcultural nursing, Migrant workers, Health disparities, Transcultural caring dynamics in nursing and healthcare model

\section{INTRODUCTION}

\subsection{Vulnerable populations' overview}

Vulnerable populations include groups having a higher likelihood of experiencing difficulties in the access of healthcare and, as a result, poorer health outcomes and higher mortality rates when compared to other groups who are less vulnerable. ${ }^{[1]}$ Vulnerability occurs when groups of people are exposed to disparate risk and health burden in comparison to other groups in the community. Marginalization of vulner- able groups occurs as a consequence of historical and current experiences and by differences alone, which may contribute to actions of discrimination. The underserved status of migrant workers is often related to economic, political, social circumstances, and dependence on others. Consequently, individuals who are underserved, marginalized, or vulnerable, are confronted with significant difficulty in accessing and using comprehensive and timely health-related services. ${ }^{[2,3]}$ Immigration is fiercely debated in political, economic, social,

\footnotetext{
${ }^{*}$ Correspondence: Denise Nagle Bailey; Email: baileyd@lasalle.edu; Address: School of Nursing, La Salle University School of Nursing and Health Sciences, Philadelphia, Pennsylvania, USA.
} 
and ethical contexts. Over $80 \%$ of undocumented individuals living in the nation are arriving from Mexico and Latin American countries including Guatemala. Most of these immigrants are vulnerable as a population, are uninsured, and not able to able to fully access the U.S. healthcare system. ${ }^{[4]}$

Migrant workers as a vulnerable population place considerable strain on the U.S. healthcare system as millions of dollars are spent annually for workers who utilize emergency departments and other hospital services. Additionally, migrant workers rely on the health care system for prenatal care and delivery of babies, and for pediatric services because of their inability to pay for care. Furthermore, the escalating costs of health care for migrant workers and their families and their inability to pay for needed services often lead to a postponement of health services. The absence of health insurance makes it difficult for migrant populations to access care, since two thirds of this population live in poverty. ${ }^{[4,5]}$

There are a number of factors placing migrant workers at risk for poor outcomes relating to health. Lack of insurance or an underinsured status is only one concern. Migrant workers are often non-English speaking and are transient in their lifestyle, working long and unpredictable hours from dawn to dusk. Low educational levels and literacy are commonly noted within this population, which contributes to the barriers they commonly encounter in the access of health care. ${ }^{[6]}$

Although the Affordable Care Act (ACA) provides the same access and requirements for health coverage to Naturalized citizens as United States-born citizens, and somewhat limited federal coverage for lawfully present immigrants, undocumented immigrants are not eligible for any benefits under the ACA. However, undocumented immigrants are eligible for emergency care under federal law and are eligible for Emergency Medicaid if they are low-income. ${ }^{[7]}$

\subsection{Backstretch workers as a vulnerable sub-population}

Migrant backstretch workers are defined as a group of itinerant laborers whose job is to care for thoroughbred horses at racetracks across the nation. ${ }^{[8]}$ Currently, there are limited studies that document a systematic analysis of the collective health issues experienced in this underserved population or how their poor health status and illnesses are related to the nature of their work and the less desirable living conditions in which they reside. ${ }^{[8]}$ Research conducted by Castaneda, Kline, and Dickey ${ }^{[8]}$ at a Florida racetrack included 84 backstretch workers in a survey to develop self-reported medical histories and worker concerns. Results from respondents in this study revealed most health issues are related to pain and musculoskeletal injury. Following these reported health

Published by Sciedu Press issues were complaints of gastrointestinal illnesses and a number of chronic conditions including hypertension and diabetes. Bailey and Dougherty's research revealed similar results after conducting a needs assessment of backstretch workers at a Pennsylvania thoroughbred racetrack. ${ }^{[5]}$ Findings indicated that common issues in this population included work-related physical injuries and chronic, noninfectious diseases including diabetes and hypertension. Other significant areas of concern identified a of a need for bilingual communication between nursing staff and clients, access to healthcare services, women's healthcare issues, and improvement in nutritional status. ${ }^{[5]}$

Housing for migrant backstretch workers on racetrack grounds is generally poor with living conditions that are sub-standard. Typically, workers come together forming small communities located behind the racetrack (backstretch). Workers engage in daily tasks related to the direct and indirect care of thoroughbred racehorses in stable-like milieus ridden with multiple environmental health and safety hazards that threaten cumulative health risks and decrease client health status. It is not uncommon for clients working at less prestigious racetracks to live in tack rooms, which are small storage rooms used for the storage of riding gear (saddles and bridles) and records that are attached to or located within stables. These inferior living conditions, along with scarce support services, contribute to the poor health status experienced by backstretch populations. Larger, more prestigious racetracks often offer backstretch workers and their families a more comprehensive selection of health services, which include medical services, health and wellness services, dormitories, schools, libraries, and churches. ${ }^{[5,9,10]}$

The horseracing industry contributes an excess of 39 billion dollars to the U.S. economy each year, and it is estimated that 4.6 million individuals are directly involved in this commercial enterprise. ${ }^{[11]}$ Individuals working within this industry are at greater risk for developing a decreased health status and ill health as a result of their repeated exposure to hazards lurking in the equine environment. ${ }^{[11]}$ The general health status of migrant backstretch workers, a sub-population working within the horseracing industry, is dependent upon the quality of the physical environment in which they work and live. Workers' encounter and experience multiple environmental hazards each day as they complete routine tasks in close proximity to the horses in stables and horse barns. Their cumulative exposure to the allergens found in hay, horse dander, saliva, and hair, increase the workers' risk of developing allergies and respiratory conditions including diseases of the airway and asthma. Other environmental hazards present in the workers environment contribute to their risk for infection, non-respiratory illness and disease, and an increased inci- 
dence of dermatological diagnoses and conditions including rashes and fungal infections. ${ }^{[9-11]}$

\subsection{Health disparities common to backstretch popula- tion}

\subsubsection{Access to health care services}

Estimates indicate there are 9.3 million undocumented individuals living in the United States. The U.S. spends an excess of $15 \%$ of its Gross Domestic Product (GDP) on healthcare each year, which accounts for more than any other developed nation in the world; however the advantages experienced by individuals using the health care system are not consistently encountered by all living in the nation. There are more than 47 million Americans that are currently without health care because of a lack of insurance. The lack of health insurance complicates the access of health services for undocumented and uninsured populations. ${ }^{[4]}$ With the initiation of the health care reform bill in the U.S. in 2009 and its subsequent enactment, many questions remain unanswered about health care for immigrants, and in particular, those who are undocumented or unauthorized. Specifically, issues relating to the health care needs of immigrants residing in the U.S. and which health care policies would yield the best outcomes for these disadvantaged groups remain unaddressed. The ongoing controversy encompassing undocumented immigrants and their health care needs continues as a public debate. ${ }^{[12]}$

\subsubsection{Women's healthcare}

Similar to their male counterparts, migrant women do not routinely seek preventive care. For migrant women, this includes routine screenings for breast and cervical cancers. Multiple factors impeding women's care relate to cultural norms, religious or spiritual beliefs, chronic poverty, lack of health insurance, transportation, knowledge deficit, confidentiality, and psychological factors including embarrassment and the fear of cancer. ${ }^{[5,13,14]}$ Language barriers and migratory patterns also are aspects that create barriers to women's health. ${ }^{[5,13,14]}$ Fear of retribution for taking time off from work to seek procedures further delay mammography and PAP screenings. This issue is compounded by the unfamiliarity of access requirements and clinic locations where preventive services are offered. ${ }^{[13]}$ The incidence and mortality rate for cervical cancer is twice as high in Hispanic women when compared to non-Hispanics. The incidence and mortality rate for breast cancer is lower in Hispanic women than non-Hispanic women however; Hispanic women are more likely to be diagnosed at a more advanced stage of the disease, lowering their survival rate and prognosis. ${ }^{[5,14]}$

Over the past decade there have been increased numbers of female migrant workers who are among the most medically underserved, least educated, and poorest ${ }^{[15]}$ of workers. Sta- tistically, one in five migrant women experience physical violence. Migrant women experience depression and suicidal ideation, acculturative stress, sadness, and loneliness more than male migrant workers and are also diagnosed more frequently than migrant men with hypercholesterolemia, hypertension and diabetes. This disparate group is not wellrepresented in the literature, leaving gaps among healthcare providers and public health personnel in understanding of and interventions for health care needs. ${ }^{[15]}$

\subsubsection{Nutritional status}

Working at the backstretch of a racetrack has been found to negatively impact workers' diets. Workers residing at racetracks experience more significant income constraints than counterparts living off campus, limiting available resources for food, emphasis on quality of nutrition, and purchasing and storing food. ${ }^{[5,8]}$ Some racetracks have on-site cafeterias or snack bars; however foods of poor nutritional quality are often served (hamburgers, French fries, pizza, sugared drinks). Convenience foods available for purchase in these venues are expensive, and the option to buy food at local markets pose additional challenges in the preparation of meals as a result of inadequate cooking accommodations which typically do not include stoves and refrigerators in workers' austere living quarters. ${ }^{[5,8]}$ Food items purchased at local markets often include items that are easy to prepare, and inexpensive to purchase; however, these items are associated with high fat and salt content, further diminishing the health status of backstretch workers. Economic considerations relating to fast food items and their consumption lead to dietary risk factors including high fat, high carbohydrate, and low nutritional value of food, and the high cost of purchasing food contributes to the vulnerability of migrant backstretch. ${ }^{[5,8]}$ In addition to the low-cost options referenced above, resident backstretch workers also opt to skip meals as an economic necessity. ${ }^{[8]}$

\subsubsection{Social justice}

Social justice propels individuals to do what is best for an individual or a group by assessing their particular needs; from a perspective that as human beings, we are collectively entitled to certain inalienable rights. ${ }^{[8]}$ Social justice infers that as a result of particular circumstances increasing an individual or group's risk, there is a reduced opportunity to advocate for self and live a quality life. Consequently, it is crucial that the actions taken by nurses and other health care providers focus on doing no harm (non-malevolence), and consequently ensure a high degree of beneficence (doing good) to all. Advocating for human rights protection and social justice needs to be acknowledged through a cultural lens which includes individuals within the context of the environment where these people live, work, and share their life with 
others. Social justice focuses on advocacy to eliminate health disparities through adherence to the basic human right of accessing quality health care. It is through the combination of basic human rights and social justice that function as a reciprocal synergy that helps ensure culturally competent care to vulnerable clients, including migrant backstretch workers, as both of these principles are upheld in clinical settings and in the broader community. ${ }^{[2]}$ For nurses, the importance of applying principles of social justice in the clinical setting and in nursing practice is predicated in the nurses increased awareness of factors (education, literacy, and socioeconomic determinants) that influence an individual's potential or capability. Social justice also differentiates between inequities and inequalities, of which, inequalities between men and women can be explained by unavoidable differences between the sexes (e.g., uterine cancer vs. prostate cancer) while inequities refer to unfair, unjust, unfair, avoidable or unnecessary differences. ${ }^{[13]}$ Migrant men and women are vulnerable to these inequalities, placing them at a higher risk for ill health.

Economic issues comprise one important area in the discussion of social justice for migrant seasonal farm workers (MSFWs) and can be generalized to the migrant backstretch population who work through cycles of unemployment and underemployment. As these individuals experience the loss or absence of work, there is an association for developing unhealthy behaviors: decreased social support; increased risk for developing psychological stress, depression, and negative mental health sequela; and cardiovascular ailments. The fear of job insecurity is intensified by workers' low educational levels and poor communication skills resulting from language barriers that often exist as an outcome of unemployment and underemployment. In their desperation to find work, migrant populations may be inclined to accept employment in jobs that discriminate, exploit, and mistreat workers. $^{[13]}$

\subsubsection{Bilingual communication and low literacy}

Spanish is the predominant language spoken at thoroughbred racetracks across the nation, and is a barrier to accessing health care services. ${ }^{[10]}$ Language barriers are problematic for both the clinician and migrant client. ${ }^{[16]}$ Conducted research in the delivery of medical care to undocumented migrants in Denmark, identifying the language barrier as an expressed concern of physicians treating migrants in the emergency room setting. Physicians commented that working with this group of individuals made their work more complex because they were not able to readily communicate the health issue or concern, making diagnosis and treatment more difficult. Conversely, it was reported that practitioners articulated a concern for the added levels of stress migrant workers experienced during a client encounter as a consequence of not understanding the medical issue, and how the communication barrier prevented the practitioner from reassuring the client using a common language. ${ }^{[16]}$ Several strategies to alleviate communication barriers include arranging for an interpreter, making use of a bilingual employee, and looking to the client's network of family, friends, or other contacts to interpret and convey health information. The use of non-verbal cues offers another viable strategy for nurses. ${ }^{[5,16]}$

The low levels of literacy of backstretch workers presents an added hurdle for nurses as they develop and disseminate health education materials, client instructions, and secure signed consents for screening and procedures. An additional problem arises for non-English speaking clients who experience low literacy levels, and need translation of health information. Presenting information using a clear and simplistic format based on the clients educational and reading levels promotes client understanding of materials, particularly if simple illustrations are included. ${ }^{[5,17]}$ Low literacy elicits negative outcomes including increased medication errors made by migrant clients, increased difficulty navigating the health care system, and a decreased likelihood that clients will be compliant in taking prescribed medications. These factors eventually result in increased emergency room visits and admissions. ${ }^{[5,17]}$

\subsection{Vulnerability of backstretch workers to specific dis- eases or conditions}

The migratory patterns typically associated with migrant populations and their reluctance to disclose illness and diagnoses, impede the tracking of health statistics and data needed to identify the numbers of workers diagnosed with chronic health conditions including hypertension, diabetes, asthma and respiratory illnesses, dermatological conditions, and other work-related ailments. ${ }^{[5,8,10]}$ Like migrant seasonal farm workers, occupational and environmental exposures to dust and airborne particles in horse barns and stables from saw dust and wood shavings used as bedding in horse stalls contribute to increased reports of respiratory and eye irritation in workers. ${ }^{[8,10]}$ The horse stables and surrounding backstretch environment where workers complete daily tasks and reside, pose cumulative health risks to migrant backstretch workers as they inhale noxious airborne endotoxins and particulates, equine allergens, ammonia, and encounter inorganic and organic dust particles. ${ }^{[9,18]}$ The occupational demands in which these workers engage is taxing upon the body, resulting in an increased the risk of musculoskeletal injuries. Acute and chronic injuries occur during the direct handling of horses and include traumatic head injuries, bites, 
contusions, and lacerations. Additional injuries commonly reported include back pain, torn rotator cuffs, repetitive stress injuries, broken ribs, and hernias. ${ }^{[8,10]}$

\section{Transcultural Care}

Although culture is a concept that can be explained through many meanings and definitions, ${ }^{[19]}$ it can be described as the set of customs, beliefs, value systems, and social structures that delineate patterns of activity and symbolic arrangements that give meaning to the behavior of human beings. ${ }^{[1]}$ Delivering culturally competent health care, with a keen awareness to the cultural aspects that influence behaviors contributing to a client's health and wellness status, is paramount to assuring quality health care to individuals, families, and communities. ${ }^{[1]}$

Madeline Leininger's seminal research in the field of transcultural caring and her Theory of Culture Care and Universality provided the requisite foundation on which the discipline of transcultural nursing was established. ${ }^{[9,19,20]}$ Leininger's theory and its espoused tenets are recognized as major influences within the nursing profession and popularity of the theorist's ethno nursing model is growing as theoretical elements are applied in nursing administration, research, practice, and education. ${ }^{[19]}$ Leininger's Theory of Culture Care and Universality ${ }^{[21]}$ embraces nurse caring through a lens that emphasizes the importance of client culture.

Transcultural caring ${ }^{[19]}$ is defined as "the relationship between charity and the right action-between love and compassion and response to suffering and need, and justice and fairness of doing what ought to be done within the (dynamics) of culture (nursing situations, organizations), or society". ${ }^{[19]}$ Contained within this definition are a number of elements relating to culture and caring: love, compassion, justice or fairness dynamics of culture, and nursing situations/organizational cultures/society. ${ }^{[19]}$ Transcultural nursing acknowledges the beliefs, values, and the attitudes of clients by considering economic, environmental, technological, and cultural perspectives. Nurses who develop an appreciation of and knowledge for multiculturalism, panethnicity (the dynamic relationship that occurs between culture and identity), cultural diversity, and are able to recognize the dynamic changes that occur over time in population groups are better prepared to provide culturally competent care in complex situations occurring across practice settings. ${ }^{[19]}$ Furthermore, it is important for nurses to recognize that an integral part of transcultural care includes an appreciation for the spiritual and religious influences that clients and their families rely on during decision-making processes relating to health and wellness, healing, or end of life decisions. ${ }^{[19]}$

\section{THEORETICAL TRANSCULTURAL FRAME- WORK}

Dr. Marilyn A. Ray's Transcultural Caring Dynamics in Nursing and Health-Care Model was developed to assist nurses in answering questions relating to the care of culturally diverse clients and the processes that impact nursing from a transcultural perspective. ${ }^{[19]}$ Four major concepts, or caring dynamics, are contained within the model: ${ }^{[19,22]}$ the essence of caring, transcultural caring ethics, transcultural context, and universal or spiritual sources. These concepts collectively provide a framework in which nurses can develop a greater understanding and awareness of different cultures and populations living in our communities and across the globe, supporting client choice through the giving and receiving culturally congruent care. ${ }^{[19,22]}$

Ray ${ }^{[19,22]}$ described and defined the essence of a caring dynamic by incorporating the multiple constructs of love, copresence (being present to others with love and compassion), availability, attendance, empathy, authenticity, communication, and compassion. Love represents a compelling energy that is needed to direct moral aspects of caring and behavior, and also frames fairness within a cultural context. The transcultural caring ethics dynamic examines the shaping of knowledge and the moral caring experiences that contribute to transcultural caring. Constructs specific to this dynamic include respect, trust, fidelity, commitment, the good, autonomy, justice, beneficence, and human rights, cultural rights, and natural rights. ${ }^{[19,22]}$ The transcultural context, takes into account "diverse identity" and the personal, ethnic, beliefs, attitudes, and cultural values individuals have in their relationships, rituals, and symbolic representations which serve as guiding forces in transcultural experiences in health care, social systems, shared or community systems, and systems of global magnitude. The construct governing this definition is the individual within the family, society, and the economical, cultural-political, technological, ecological, and health care systems that are in place. ${ }^{[19,22]}$ Universal sources include prevailing religious and spiritual traditions and rituals that influence transcultural caring experiences that are built on religion and spirituality. These sources include "God, Christ, Buddha, the Absolute, Allah, Power, Force, Energy, Creativity, Healing and Mythology". [19] The four caring dynamics come together at the center of Ray's model ${ }^{[19]}$ to assist nurses in the development of transcultural caring awareness and understanding' creating a foundation for nurses to care for the transcultural health care needs of clients. ${ }^{[19,22]}$

Transcultural caring is central to the discussion of the migrant backstretch worker as a vulnerable and marginalized sub-population. Ray ${ }^{[19]}$ defined transcultural caring as "the 
relationship between clarity and the right action-between love and compassion and response to suffering and need, and justice and fairness of doing what ought to be done within the dynamics of culture, or society". [19]

Understanding transcultural consciousness and acquiring a sensitivity to the importance of developing a transcultural framework for practice is critical for not only the community nurse, but for all nurses in their respective professional roles. Nurses who develop transcultural awareness also have a heightened appreciation for diversity and share in in a "common humanity". [19]

\section{ROLES OF CLINIC NURSES AND STAFF}

The La Salle Neighborhood Nursing Center (LSNNC) is a nurse-led health and wellness center that has been in operation since 1991. LSNNC serves a multi-cultural and diverse urban population who are at-risk for poor health and live in the community. The LSNNC implements a population health approach in congruence with La Salle University's mission and vision to provide quality in-house and community outreach programs designed to enhance and support client health Operational goals of LSNNC are accomplished as faculty, staff, and nursing students lead health education and wellness activities, engage clients in health promotion and disease prevention strategies, screen and immunize clients, and work with clients who are in need of a referral for primary or specialist care. ${ }^{[23]}$

For 17 years, LSNNC has been actively involved in a health and wellness program for migrant backstretch workers at Philadelphia Parx Racetrack. The resident chaplain at the racetrack initially invited LSNNC to provide health and wellness services to the men and women working at the backstretch of the racetrack. Funded by the Pennsylvania Thoroughbred Horsemen's Association, nursing faculty, and student nurses, staff (including an AmeriCorps worker) promote heath through education, screening, counseling, case management, and referral. Screenings offered include blood pressure, cholesterol, and glucose. Immunizations (influenza, hepatitis B, tetanus, and pneumonia,) are also available to workers. Weekly clinic hours are scheduled at the backstretch clinic to serve this underserved population. There is an onsite physician (part-time) who can be contacted if a client requires further evaluation and/or diagnostic testing. ${ }^{\text {[23] }}$

During the 2013-2014 academic year, LSNNC faculty, nurses and nursing students working at the backstretch clinic reported a total of 485 client encounters. Nurses administered 129 influenza, tetanus, and pneumonia vaccines, and functioning as a Level III licensed clinical laboratory, performed 13 blood glucose screenings. Blood pressure screenings were available on a weekly basis and totaled 473. Health and wellness education sessions were attended by 164 clients and covered topics related to health promotion and disease prevention, sick care, care of injury, use of medications, and breast health. Other nursing services available at the clinic include health assessments, case management, and referrals. Client referral sources include Health Link, Bucks County Health Improvement Partnership (BCHIP), Healthy Women 50 Plus (state funded mammography), county mental health services, and the ACA for eligible individuals. If appropriate, nurses and staff serve as navigators, directing insured workers to appropriate out-of-state insurance carriers.

\section{NURSE-PATIENT AGREEMENT WITH WOR- KERS}

The LSNNC adheres to a strict practice of securing a signed Client and Information Form prior to the provision of services at the backstretch clinic. This form is designed for purposes of obtaining client signatures for health and screening services, follow-up for additional care if needed, and also permits nurses to contact a medical provider if clients have one. Client health information is confidential and kept in a locked file. Aggregate client data pertaining to screening results are recorded and clients are made aware that the results of these services may be studied to improve health care delivery of individual clients and others residing in the backstretch community. Clients also receive a copy of the Patient Bill of Rights and Responsibilities. Both documents are discussed with clients prior to the commencement of clinical services.

\section{DISCUSSION}

\subsection{Nursing interventions}

Nursing at the backstretch clinic involves creative program planning strategies that encompass practical objectives to enhance the health status of all clients receiving clinical services. Emphasis placed on offering basic health interventions through assessment and evaluation of clients using an integrative approach and incorporating goals that are culturally congruent, client-centered, realistic, and achievable for this disenfranchised and unique population. ${ }^{[5]}$ Clinic nurses and staff are collectively mobilized in efforts to maintain goals that focus on the improvement of the client's basic life skills and the development of positive health behaviors. Furthermore, nurses, staff, and nursing students reinforce disseminated health and wellness information relating to client health issues; this is achieved through active follow-up of clients identified with abnormal screening results, medicine adherence issues, and other health and wellness concerns (e.g., nutritional counseling for diabetic or obese clients). 
Client referrals to the on-site physician and calls to agencies and clinics in the community provide some additional support to migrant backstretch clients. These efforts continue as active components of the case management process; however, this is an area that needs to be expanded in order to yield more robust options for clients. ${ }^{[5]}$ Health information is available in Spanish and English to assist clients; literacy remains an issue. The addition of bilingual interpreters might improve client communication of health information and understanding of emergent health issues. Language barriers and the limited capabilities of faculty, staff, and nursing students to translate English to Spanish continues to present a barrier to health care considering the primary language of the backstretch population is Spanish. ${ }^{[5,10]}$

Delivering culturally competent nursing care to workers at the backstretch clinic remains a priority to alleviate health disparities experienced by migrant workers. Empowering clients by including realistic strategies that promote and improve health through the integration of cultural beliefs and folk practices into nursing care plans and wellness programs, contributes to overall client health and has relevance in the area of expanded access to health care services for these marginalized individuals. ${ }^{[24]}$

Faculty, nursing students, and staff take into account the cultural differences of migrant clients and center efforts on understanding and respecting different points of view that are present within the backstretch population, placing increased consideration on these differences. One example includes caring for clients who feel they are not in control of their diabetes. In this instance, more emphasis is placed on client nutrition and diabetes education. ${ }^{[25]}$ Nurses also provide services at the clinic within the context of the workers' cultural habits and formulate care plans that target individual client differences. Transcultural nursing is an integral part of nurse practice. The first step in developing cultural competency is predicated upon the nurse's desire to become culturally competent which progresses in a trajectory toward acquiring the requisite knowledge to understand the transcultural process. ${ }^{[25]}$

\subsection{Teaching students Ray's model}

Although the theoretical model and tools developed by Dr. Ray in her Transcultural Caring Dynamics in Nursing and Health Care model are pivotal to understanding the complex and intricate processes required to reach more advanced or sophisticated levels of transcultural competency and transcultural nurse caring, the scope and context of this research. One plausible intervention gleaned from the model that would enrich the current undergraduate clinical curriculum and improve the delivery of culturally competent care to back- stretch workers entails operationalizing Ray's Transcultural Caring-Based Learning Approach (TCCBL) in pre and post conference discussions. Application of the TCCBL learning approach would imbue a framework to assist faculty and students in their collaborative efforts to create a culturally dynamic community within the backstretch milieu. ${ }^{[19]}$

The Transcultural Caring-Based Learning approach ${ }^{[19]}$ was developed using the problem-based learning (PBL) and continuing competence model currently in use at a number of medical and nursing schools in North America. ${ }^{[19]}$ TCCBLs utility and applicability to small working groups of 2 to 12 students would be optimum for inclusion in the clinical setting. Forming student groups with a nursing instructor who facilitates and cocreates ${ }^{[19]}$ through an interjection of personal understanding in conversations with nursing students could assist students as they participate in transcultural dialogue. This conversation can be predicated upon critical caring inquiry (reflection) and is integrated with materials presented during class lecture that emphasize working with culturally diverse and vulnerable populations, and transcultural caring theory. Complementing strategies augmenting this strategy would require students to gather evidence-based literature, and actively explore Internet sources (data and other forms of media), to unearth transcultural information. The TCCLB process can be viewed as a means to empower students to collect transcultural evidence through a lens of critical caring inquiry that incorporates reflection and "transcultural communicative spiritual-ethical caring" using a pattern identification process that would include pattern "seeing", pattern mapping, pattern recognition, and pattern transformance. ${ }^{[19]}$

Incorporating key theoretical elements espoused in the Transcultural Caring Dynamics in Nursing and Health Care model, ${ }^{[19]}$ in conjunction with the addition of Ray's TCCLB learning approach, might function as a useful tool for nursing students as they develop and improve transcultural competencies during their transcultural experiences with migrant clients. The TCCLB approach used within a context of critical inquiry and reflection in what Ray describes as a "communicative spiritual-ethical caring action", integrates a pattern-identification methodology designed to actively engage nursing students in the transcultural process. ${ }^{[19]}$

During the learning process, it is critical for student nurses to be challenged to consider the cultural backgrounds of clients and the unique differences that are present in beliefs, attitudes, values, and opinions that are related to these differences and how clients perceive and give meaning to the world in which they live. Nurses also are affected by their own diverse world-views in their professional practice and private 
lives. Engaging nursing students in the Transcultural Caring Learning-Based Learning process would enable students to begin transcultural conversation by decreasing conflicts that might surface as a result of individual cultural ideologies and opinions. ${ }^{[19]}$

\subsection{Clinical nursing implications}

Improving clinical practice for nursing faculty, nursing students, and staff requires a comprehensive educational approach that offers a curriculum targeted to increase the clinician's understanding of transcultural care and cultural awareness. Understanding transcultural care and adding its components to current health and wellness programs (program planning) along with the addition of Ray's theoretical principles, can improve the overall health and well-being of migrant backstretch workers. This can be accomplished by advancing a robust curriculum that incorporates principles of transcultural care and evidence-based guidelines to enhance the quality of nursing care for clients with cultural differences. ${ }^{[26]}$ Moreover, providing clients with health information that is both culturally competent and congruent, while focusing on their educational levels and language requirements, is a significant factor for optimum delivery of meaningful health programs for workers. ${ }^{[5,28]}$ Improving the health-seeking behaviors of workers through implementation of culturally sensitive health programs serves as a viable strategy to improve the general health status of migrant clients by establishing a relationship of trust between clinicians and clients. Additionally, this critical support would function as a strategy to increase the number of weekly client encounters at the backstretch clinic for emergent issues, and improve client compliance in keeping scheduled follow-up appointments for abnormal screenings and medication compliance checks. ${ }^{[5,27,28]}$

\subsection{Challenges}

There is a gap in the extant literature that explains interventions in the workplace for vulnerable individuals relative to cost effective outcomes. ${ }^{[28]}$ Developing and implementing a health and wellness program for migrant backstretch workers is a daunting proposition for university faculty as they develop and revise baccalaureate and graduate nursing curricula to better address the unique health issues and cultural differences of marginalized individuals. Barriers impeding quality care include access, cultural disparities, social isolation, language and communication difficulties, underinsured or uninsured status, dissimilar perspectives of illness and treatment, negative viewpoints among staff and clients, and access to previous medical records. ${ }^{[5,8,27]}$ The availability of resources (financial and workforce) needed to sustain and expand services at the backstretch clinic are contingent upon

Published by Sciedu Press continued support from the Pennsylvania Horsemen's Association, LSNNC nurses, and the AmeriCorps worker, who work collaboratively in the clinic setting. University nursing faculty, along with undergraduate and graduate students assigned at the backstretch clinic, are instrumental in operationalizing health education and wellness programs, and screenings (blood pressure, glucose, cholesterol, and immunizations); however, due to the academic calendar, there are unavoidable interruptions in the continuity of health and wellness programs. On-site medical care is offered by a retired physician who evaluates migrant clients one morning each week at no cost; increasing primary care services at the on-site clinic location is needed to address the complex health issues experienced by this group of marginalized clients. ${ }^{[5]}$ Promoting and improving community engagement and building coalitions in the community with other agencies and groups is critical to meet the urgent needs of this group of itinerant laborers; current efforts at the backstretch clinic requires additional attention directed at increasing the scope of interdisciplinary health referral services within the community. ${ }^{[5,10]}$ Efforts to engage the local primary care system (physicians and nurse practitioners) at off-site locations may be significant in providing a higher level of care to workers requiring follow-up diagnostic services, screenings, or specialist referral. Meeting this challenge might significantly improve the continuity of care for underserved workers. ${ }^{[5,26]}$ Nurses working with vulnerable populations can also bring a heightened awareness to this disparate group of individuals through scholarly efforts, and also by sharing research relating to the specialized needs of migrant populations in the classroom, and at professional meetings and conferences. ${ }^{[10]}$

There are no established guidelines relating to the provision of culturally competent care that can be applied across all cultures. This represents a significant challenge as health care organizations world-wide define care based upon their unique perspectives of cultural norms that encompass economic, political, and established social systems. The variance among cultural norms and the distinctive milieus in which nurse's practice require carefully prescribed guidelines that are thoughtfully crafted for each clinical setting. ${ }^{[24]}$

\section{Conclusion}

The vulnerability of migrant backstretch workers as a marginalized sub-population in our communities is concerning as these itinerant laborers struggle with a myriad of health, economic, and social issues. Their inability to access healthcare and other vital community services and resources as a result of their invisible status in the community, and the low wages they earn contributes to the health disparities this population experiences. ${ }^{[10]}$ 
When delivering optimum health care to vulnerable populations, including migrant backstretch workers, it is crucial for nurses to develop an optimal healing environment for clients in institutional settings by establishing a caring atmosphere that transcends physical space through the creation of a sanctuary where health, wellness, and healing can occur between the client and care-giver incorporating a holistic approach. ${ }^{[29]}$ This strategy can also be applied in the clinical setting to benefit vulnerable populations, including migrant backstretch workers who live and work in environmental conditions that threaten their overall health and well-being.

Embracing and incorporating a transcultural approach into current health care practices in outpatient migrant clinics for the underserved represents one plausible strategy to enhance the overall health status and ease the spiritual suffering of clients. These marginalized individuals suffer from many diseases and conditions that are a direct result of sub-standard working and living conditions which increase their risk of developing poor health and illness. ${ }^{[5]}$ Dr. Marilyn A. Ray's Transcultural Caring Dynamics in Nursing and Health-Care Model is regarded as a useful tool for the clinician to implement into nursing curriculums and clinical practices to improve the general health of clients as faculty, nurses, nursing students, and staff work cooperatively and collaboratively to deliver care to workers. ${ }^{[19]}$ Application of Ray's theoretical components ${ }^{[19]}$ at the backstretch clinic is designed to increase clients' sense of being cared for, protected, and loved, which in turn, is expected to heighten our clients' sense of belonging in the world. ${ }^{[19]}$

\section{REFERENCES}

[1] Roux G, Halstead J. Issues and trends in Nursing: Essential knowledge for today and tomorrow. Sudbury MA: Jones and Bartlett; 2009. $322 \mathrm{p}$.

[2] Paquiao DF. Nursing care of vulnerable populations using a framework of cultural competence, social justice, and human rights. Contecmporary Nurse. 2008; 28(1-2): 189-197. http://dx.doi.org /10.5172/conu.673.28.1-2.189

[3] Williams D, Neville S. Culturally safe research with vulnerable populations. Contemporary Nurse. 2009; 33(1): 69-79. http://dx.d oi.org/10.5172/conu.33.1.69

[4] Clark P, Surry L, Contino K. Healthcare access for migrant farmworkers: A paradigm for better health. Internet Journal of Health. 2009; 8(2): 7.

[5] Bailey DN, Dougherty A. A nurse-led wellness program for migrant backstretch workers. Nursing Forum. 2013; 49(1): 1-9. http: //dx.doi.org/10.1111/nuf. 12048

[6] Anthony M, Williams J, Avery A. Health needs of migrant and seasonal farmworkers. Journal of Community Health Nursing. 2008; 25: 153-160. PMID:18709576. http://dx.doi.org/10.1080/0 7370010802221768

[7] NILC: Immigranst and the Affordable Care Act [Internet]. Los Angeles: National Immigration Law Center [cited 2014]. Available from: http://wwww.nilc.org/immigrantshcr.html

[8] Castaneda H, Kline N, Dickey N. Health concerns of migrant backstretch workers at horse Racetracks. Journal of Healthcare for the Poor and underserved. 2010; 21(2): 489-503. PMID:20453352. http://dx.doi.org/10.1353/hpu.0.0300

[9] Bailey DN, Elliott R. Living in the shadows of humanity: Environmental respiratory threats to Migrant Backstretch Worker's employed at thoroughbred racetracks. International Journal for Human Caring. 2014; 18(2): 25-32.

[10] Castaneda H. Medicine on the backstretch: Migrant racetrack workers. Streamline: the Migrant Health News Source. Migrant Clinicians Network. 2011; 17(1): 1-8.

[11] Mazan MR, Svatek J, Maranda L, et al. Questionnaire assessment of airway disease symptoms in equine barn personnel. Journal of Occupational Medicine. 2009; 59(4): 220-225. PMID:19223434. http://dx.doi.org/10.1093/occmed/kqp003
[12] Holmes SM. The clinical gaze in the practice of migrant health: Mexican migrants in the United States. Social Science in Medicine. 2012; 74(6): 873-881. PMID:21992736. http://dx.doi.org/10.1016 /j.socscimed.2011.06.067

[13] Clingerman E. Social justice: A framework of culturally competent care. Journal of Transcultural Nursing. 2011; 22(4): 334-341. PMID:21844247. http://dx.doi.org/10.1177/10436596114 14185

[14] Fernandez M, Gonzalez A, Tortolero-Luna G, et al. Effectiveness of Cultivando La Salud: A breast and cervical cancer screening promotion program for low-income Hispanic Women. American Journal of Public Health. 2009; 99: 936-943. PMID:19299678. http://dx.doi.org/10.2105/AJPH. 2008.136713

[15] Clingerman E. An insider/outsider team approach in research with migrant farmworker women. Family and Community Health/supplement. 2007; 1: S75-S84.

[16] Jenson NK, Norredam M, Draebeln T, et al. Providing medical care for undocumented migrants in Denmark: What are the challenges for health professionals? BMC Health Services Research. 2011; 11(1): 154-164. PMID:21711562. http://dx.doi.org/10.1186 /1472-6963-11-154

[17] Fleming JM. Health literacy. RDH. 2007; 27(3): 44-48, 50, 52, 54.

[18] Elfman L, Riihimaki M, Pringle J, et al. Influence of horse stable environment on human airways. Journal of Occupational Medicine and Toxicology. [Internet] 2009. http://dx.doi.org/10.1186 /1745-6673-4-10

[19] Ray MA. Transcultural nursing dynamics in nursing and healthcare. Philadelphia, PA: F.A. Davis; 2010. 320p.

[20] Bailey DN. Caring defined: A comparison and analysis. International Journal for Human Caring. 2009; 13(1): 16-31.

[21] Leininger M. Overview of the Theory of culture care with the ethnonursing research method. Journal of Transcultural Nursing. 1997; 8(2): 32-52. PMID:9369663. http://dx.doi.org/10.1177/104 365969700800205

[22] Morris EJ. Book Review: Transcultural caring dynamics in nursing and healthcare. Journal of Transcultural Nursing. 2012; 23(2): 215-216. http://dx.doi.org/10.1177/1043659611434063

[23] Lasalle Neighborhood Nursing Center [Internet] Philadelphia: La Salle University School of Nursing and Health Sciences; [cited 2014] 
Available from: http://www. lasalle.edu/academ/nursing/n _nursing/index.htm

[24] Douglas MK, Rosenkoetter M, Pacquiao DF, et al. Guidelines for implementing culturally competent nursing care. Journal of Transcultural Nursing. 2014; 25(2): 109-121. PMID:24549502. http: //dx.doi.org/10.1177/1043659614520998

[25] Maier-Lorentz MM. Transcultural nursing: Its importance in nursing practice. Journal of Cultural Diversity. 2008; 15(1): 37-43. PMID:19172978.

[26] Pottie K, Batista R, Mayhew M, et al. Improving delivery of primary care for vulnerable migrants: Delphie consensus to prioritize innovative practice strategies. Canadian Family Physician. 2014; 60(1): $32-40$
[27] Priebe S, Sandhu S, Dias S, et al. Good practice in healthcare for migrants: Views and experience of care professionals om 16 European countries. BMC Public Health. 2011; 11(1): 187-199. PMID:21439059. http://dx.doi.org/10.1186/1471-2458-1 $1-187$

[28] Zarate-Abbott P, Gilliland L, Allwein D, et al. Workplace health promotion: Strategies for low income Hispanic immigrant women. AAOHN Journal. 2008; 56(5): 217-222. PMID:18578188. http: //dx.doi.org/10.3928/08910162-20080501-01

[29] Wilson M, Casterline G. Using Caring-science to design a healing environment for transradial catheterization recovery. International Journal for Human Caring. 2013; 17(2): 50-58. 